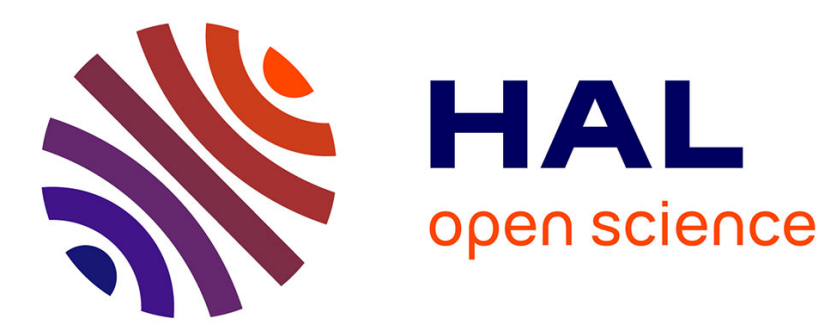

\title{
Dissipative time-domain one-dimensional model for viscothermal acoustic propagation in wind instruments.
}

Alexis Thibault, Juliette Chabassier

\section{To cite this version:}

Alexis Thibault, Juliette Chabassier. Dissipative time-domain one-dimensional model for viscothermal acoustic propagation in wind instruments.. Journal of the Acoustical Society of America, 2021, 150 (2), pp.1165-1175. 10.1121/10.0005537 . hal-03328715

\section{HAL Id: hal-03328715 https://hal.science/hal-03328715}

Submitted on 30 Aug 2021

HAL is a multi-disciplinary open access archive for the deposit and dissemination of scientific research documents, whether they are published or not. The documents may come from teaching and research institutions in France or abroad, or from public or private research centers.
L'archive ouverte pluridisciplinaire HAL, est destinée au dépôt et à la diffusion de documents scientifiques de niveau recherche, publiés ou non, émanant des établissements d'enseignement et de recherche français ou étrangers, des laboratoires publics ou privés. 
Dissipative time-domain 1D model for viscothermal acoustic propagation in wind instruments.

Alexis Thibault ${ }^{\mathrm{a})}$ and Juliette Chabassier

Project team MAKUTU, Inria Bordeaux Sud-Ouest Research Center, 200 Avenue de la Vieille Tour, 33405 Talence, France

Laboratoire de Mathématiques et de leurs Applications,

Université de Pau et des Pays de l'Adour, Avenue de l'Université, 64013 Pau,

France 
Accurate modeling of acoustic propagation in tubes of varying cross-section in musical acoustics must include the effect of viscous and thermal boundary layers. Models of viscothermal losses are classically written in the frequency domain. An approximate time-domain model is proposed, where all the physical parameters of the instrument as the bore shape or the wave celerity are explicit coefficients. The model depends on absolute tabulated constants which only reflect that the pipe is axisymmetric. It can be seen as a telegrapher's equations augmented by an adjustable number of auxiliary unknowns. A global energy is dissipated. A time discretization based on variational approximation is proposed along with numerical experiments and comparisons with other models.

a) alexis.thibault@inria.fr 
Viscothermal time model for wind instruments

\section{INTRODUCTION}

Time-domain simulations of wind musical instruments require the choice of a balance between efficiency and accuracy. Real-time synthesis techniques, such as digital waveguide synthesis with lumped wall loss filter (Abel et al., 2003), (Mignot et al., 2010), modal decomposition (Silva et al., 2014), and Finite Difference - Time Domain (Bilbao and Harrison, 2016), favor their performance objective at the expense of model and discretization errors. By contrast, computer aided instrument prototyping requires a fine assessment of the physical phenomena occurring in the instrument, including dissipation and dispersion effects on propagative waves inside air filled pipes, caused by viscous and thermal boundary layers. The Navier-Stokes equations of fluid dynamics indeed do account for all these effects, but a practical understanding of the macroscopic phenomena from direct numerical simulation requires a tremendous computational effort. In the different characteristic regimes found in musical instruments, several approximate 3D or 1D models exhibit good accuracy and performance ; they are briefly reviewed thereafter. More details about the qualitative 
and quantitative fulfilment of their underlying assumptions can be found in (Thibault and Chabassier, 2020). Most of them, including the prevalent model initially derived by (Zwikker and Kosten, 1949) (ZK), are naturally expressed in the frequency domain, which hampers their use for synthesising the sound of musical instruments by coupling the pipe to a nonlinear embouchure and using time-varying commands. This work focuses on the time domain formulation of a stable and accurate 1D model, and its numerical implementation based on a variational approach, aiming at computer aided instrument prototyping.

\section{A. Viscous and thermal boundary layers in 3D}

The Linearized Navier Stokes (LNS) equations, also called thermoviscoacoustic equations, describe the evolution of the acoustic density, pressure, temperature and velocity, which are small perturbations of a background medium assumed steady with no mean flow. They feature acoustic wave propagation, as well as viscous and thermal diffusion (Tijdeman, 1975).

For a domain filled with air bounded with rigid, non-porous isothermal walls, and when the acoustic wavelength is greater than the viscous and thermal characteristic lengths, the acoustic velocity and temperature are functions of the acoustic pressure and two potentials (Kampinga et al., 2011), which are solution to non homogeneous heat equations with Dirich- 
Viscothermal time model for wind instruments

\section{B. Model reduction to $1 \mathrm{D}$}

In the case of a cylindrical pipe, (Kirchhoff, 1868) derives an implicit analytical dispersion relation to the LNS equations that must be solved iteratively, which does not offer a practical modeling framework. The reduced 1D model derived from an approximation of LNS by (Zwikker and Kosten, 1949) has been shown to be valid for audible frequencies and assuming the reduced frequency $k=\omega R / c$ is small, where $\omega$ is the angular frequency, $R$ the radius of the pipe and $c$ the celerity of the wave (Tijdeman, 1975). More precisely, the average pressure along a pipe section $\hat{p}(x, \omega)$, and the air flow $\hat{v}(x, \omega)$, along the longitudinal spatial 
variable $x \in[0, L]$, are solution to the so-called Zwikker and Kosten (ZK) model:

$$
(\mathrm{ZK})\left\{\begin{array}{l}
\frac{d \hat{p}}{d x}+j \omega \frac{\rho}{S} \frac{1}{1-F_{v}(\omega)} \hat{v}=0 \\
\frac{d \hat{v}}{d x}+j \omega \frac{S}{\rho c^{2}}\left[1+(\gamma-1) F_{\theta}(\omega)\right] \hat{p}=0
\end{array}\right.
$$

where $S$ is the pipe section, $L$ its length, $\rho$ the static density, $\gamma$ the ideal gas constant, and coefficients $F_{v}$ and $F_{\theta}$ describe the contribution of respectively viscous and thermal dissipation, as:

$$
F_{v}(\omega)=\phi\left(R \sqrt{-\frac{j \omega \rho}{\mu}}\right), F_{\theta}(\omega)=\phi\left(R \sqrt{-\frac{j \omega \rho C_{P}}{\kappa}}\right)
$$

$$
\text { with } \phi(\alpha)=\frac{2 J_{1}(\alpha)}{\alpha J_{0}(\alpha)}
$$

where $\mu$ is the gas viscosity, $\kappa$ the thermal conductivity, $C_{p}$ the specific heat with constant pressure (see Table I), and where $J_{0}$ and $J_{1}$ are zeroth- and first-order Bessel functions.

Although it has been derived in the context of cylindrical geometries, model (ZK) has been used intensively for varying geometries, namely for $S$ depending on the longitudinal variable $x$. The classical horn equations describing plane wave propagation in an axisymmetric lossless pipe can be retrieved from an asymptotic analysis relying on Euler's equations in a pipe with varying section (Rienstra, 2005). Model (ZK) can be seen as a perturbation of these horn equations, and has been employed for dissipative pipes with varying section for instance in (Chaigne and Kergomard, 2016), (Bilbao and Harrison, 2016), (Tournemenne 


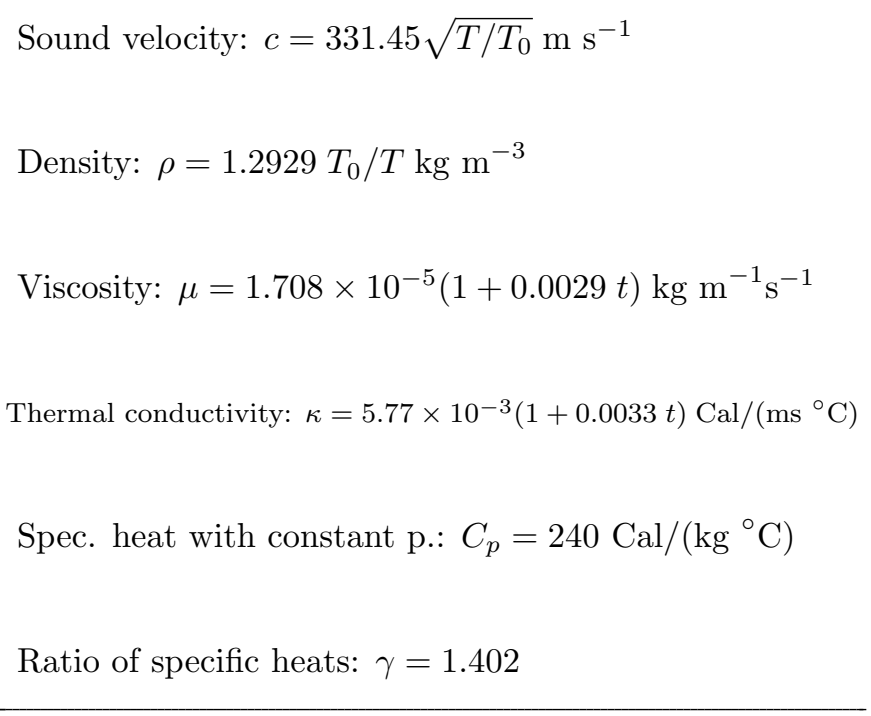

TABLE I. Numerical values (Chaigne and Kergomard, 2016) of air constants used in the model. $t$ is the temperature in Celsius, and $T$ the absolute temperature with $T_{0}=273.15 \mathrm{~K}$.

and Chabassier, 2019) in the harmonic regime. Curvature of the wave fronts can occur in varying geometries and especially in the instrument bell, which can be modeled by an equation similar to (1) (Hélie et al., 2013). The present work focuses on viscothermal effects, and will neglect the curvature effects which may be included in future work.

The highly nonlinear dependency of $F_{v}$ and $F_{\theta}$ with $\omega$ induces a nonlocal formulation in the time domain leading to mathematical and numerical intricacy, which motivates the present work. 


\section{Model approximations and time domain representations}

Model (ZK) has been approximated for different frequency regimes and/or pipe sizes,

especially when the Stokes number $s=R \sqrt{\rho \omega / \mu}$ is large (Tijdeman, 1975), (Keefe, 1984), (Stinson, 1991), (Scheichl, 2004). Terms in $\sqrt{j \omega}$ arise in the derived equations, leading in the time domain to fractional derivatives. Note that similar terms arise in the Webster-Lokshin 1D model which models the acoustic pressure close to the boundary layers using Cremer 3D effective wall impedance (Hélie et al., 2013). These terms can be treated numerically with approximations of diffusive representations (Hélie and Matignon, 2006), (Berjamin et al., 2017). Other approaches are based on direct diffusive representations of model (ZK) (Thompson et al., 2014), (Bilbao and Harrison, 2016), (Schmutzhard et al., 2017).

\section{Contribution and outline}

One important aspect of space and time discretization of a model is to control the trade-off between accuracy and efficiency, in all targeted applications and configurations.

In the context of computer aided instrument prototyping, our purpose is to ensure numerical stability and quantify accuracy with respect to model (ZK). Energy-based methods have 
Viscothermal time model for wind instruments

proven especially efficient to discretize wave equations in time (Van Der Schaft, 2006),(Bilbao, 2009), (Cohen, 2013), (Hélie and Silva, 2017), (Chabassier et al., 2020) (Chatziioannou, 2019). The model of (Bilbao and Harrison, 2016), which satisfies an energy balance identity, is particularly suitable for physics-based sound synthesis. However, synthetizing sounds of a specific instrument with this model requires to run an optimization algorithm for every different value taken by the pipe radius and temperature before running time iterations. The present work makes this optimization step geometry-independent, by re-writing the model of (Bilbao and Harrison, 2016) with a new expression of the coefficients. Optimized constants are given in Table II and are usable directly in synthesis algorithms. The new coefficients of the model explicitly depend on the non-constant geometrical and physical parameters, therefore no optimization must be implemented nor launched before time iterations.

The model, presented in Sec. II along with its energy balance, is suitable for variational approximation, therefore naturally compatible for coupling with other energy-based models. Its mathematical derivation is developed in Sec. III and a practical implementation method is proposed in Sec. IV along with a space and time discretization which guarantees a discrete energy identity and an algorithmic strategy for explicit update of the unknowns. A numerical scheme using 1D mixed spectral finite elements is proposed, allowing high-order space 
Viscothermal time model for wind instruments

\section{MAIN RESULT}

The acoustic pressure averaged on a pipe section and the volume flow can be modeled by the solutions $p(x, t)$ and $v(x, t)$ to the following system for $x \in] 0, L[, t>0$, where $N$ is an 
integer and $v_{i}(x, t), p_{0}(x, t), p_{i}(x, t)$ are $2 N+1$ auxiliary variables:

$$
\left\{\begin{array}{l}
\frac{\rho}{S} \frac{\partial v}{\partial t}+R_{0} v+\sum_{i=1}^{N} R_{i}\left(v-v_{i}\right)+\frac{\partial p}{\partial x}=0, \\
\frac{S}{\rho c^{2}} \frac{\partial p}{\partial t}+G_{0}\left(p-p_{0}\right)+\sum_{i=1}^{N} G_{i}\left(p-p_{0}-p_{i}\right) \\
\quad+\frac{\partial v}{\partial x}=0, \\
L_{i} \frac{\mathrm{d} v_{i}}{\mathrm{~d} t}=R_{i}\left(v-v_{i}\right), \quad \text { for } 1 \leq i \leq N, \\
C_{0} \frac{\mathrm{d} p_{0}}{\mathrm{~d} t}=G_{0}\left(p-p_{0}\right) \quad+\sum_{i=1}^{N} G_{i}\left(p-p_{0}-p_{i}\right), \\
C_{i} \frac{\mathrm{d} p_{i}}{\mathrm{~d} t}=G_{i}\left(p-p_{0}-p_{i}\right) \quad \text { for } 1 \leq i \leq N .
\end{array}\right.
$$

The coefficients of this system are defined as

$$
\left\{\begin{array}{l}
R_{0}(x)=\frac{\pi \mu}{S(x)^{2}} a_{0} \\
L_{i}(x)=\frac{\rho}{S(x)} a_{i}, R_{i}(x)=\frac{\pi \mu}{S(x)^{2}} \frac{a_{i}}{b_{i}} \\
C_{0}(x)=\frac{S(x)(\gamma-1)}{\rho c^{2}}, G_{0}=\frac{\pi \kappa(\gamma-1)}{\rho^{2} c^{2} C_{P}} a_{0} \\
C_{i}(x)=\frac{S(x)(\gamma-1)}{\rho c^{2}} a_{i}, G_{i}=\frac{\pi \kappa(\gamma-1)}{\rho^{2} c^{2} C_{P}} \frac{a_{i}}{b_{i}}
\end{array}\right.
$$

where the coefficients $a_{i}$ and $b_{i}$ are dimensionless constants obtained from an optimization procedure described in Section III, see Table II for $N \in\{2,4,8\}$ and supplementary material SuppPub1.txt up to $\mathrm{N}=16^{1}$. We expect that increasing the value of $N$ will make the solution closer to the original model (1), although it will also increase the number of auxiliary unknowns. The form of (3) is the same as the system used in (Bilbao and Harrison, 2016), but the values of the coefficients change, because the variable $v$ represents here the acoustic 
flow. To conform to their notations, our formulas for $R_{i}$ and $L_{i}$ should be multiplied by $S$, and $C_{i}$ and $G_{i}$ should be divided by $S$.

This model is shown in Section III to satisfy the following energy balance identity

$$
\begin{gathered}
\frac{\mathrm{d}}{\mathrm{d} t} \mathcal{E}=-Q-p(L) v(L)+p(0) v(0), \\
\mathcal{E}=\frac{1}{2} \int_{\Omega}\left[\frac{\rho}{S} v^{2}+\frac{S}{\rho c^{2}} p^{2}+C_{0} p_{0}^{2}\right. \\
Q=\int_{\Omega}\left[R_{0} v^{2}+\sum_{i=1}^{N} R_{i}\left(v-v_{i}\right)^{2}+G_{0}\left(p-p_{0}\right)^{2}\right. \\
\left.+C_{i=1} p_{i}^{2}+\sum_{i=1}^{N} L_{i} v_{i}^{2}\right] \\
\left.+\sum_{i=1}^{N} G_{i}\left(p-p_{0}-p_{i}\right)^{2}\right] \geq 0
\end{gathered}
$$

The model hence dissipates energy through the negative term $-Q$, and can exchange work with other systems via its boundaries $x=0$ and $x=L$.

\section{MATHEMATICAL DERIVATION}

System (1) can be written as

$$
\left\{\begin{array}{l}
\frac{d \hat{p}}{d x}+\frac{\rho}{S}\left[j \omega+\frac{G\left(\tau_{v} \omega\right)}{\tau_{v}}\right] \hat{v}=0 \\
\frac{d \hat{v}}{d x}+\frac{S}{\rho c^{2}}\left[j \omega+\frac{\gamma-1}{\frac{1}{j \omega}+\frac{\tau_{\theta}}{G\left(\tau_{\theta} \omega\right)}}\right] \hat{p}=0,
\end{array}\right.
$$


Viscothermal time model for wind instruments

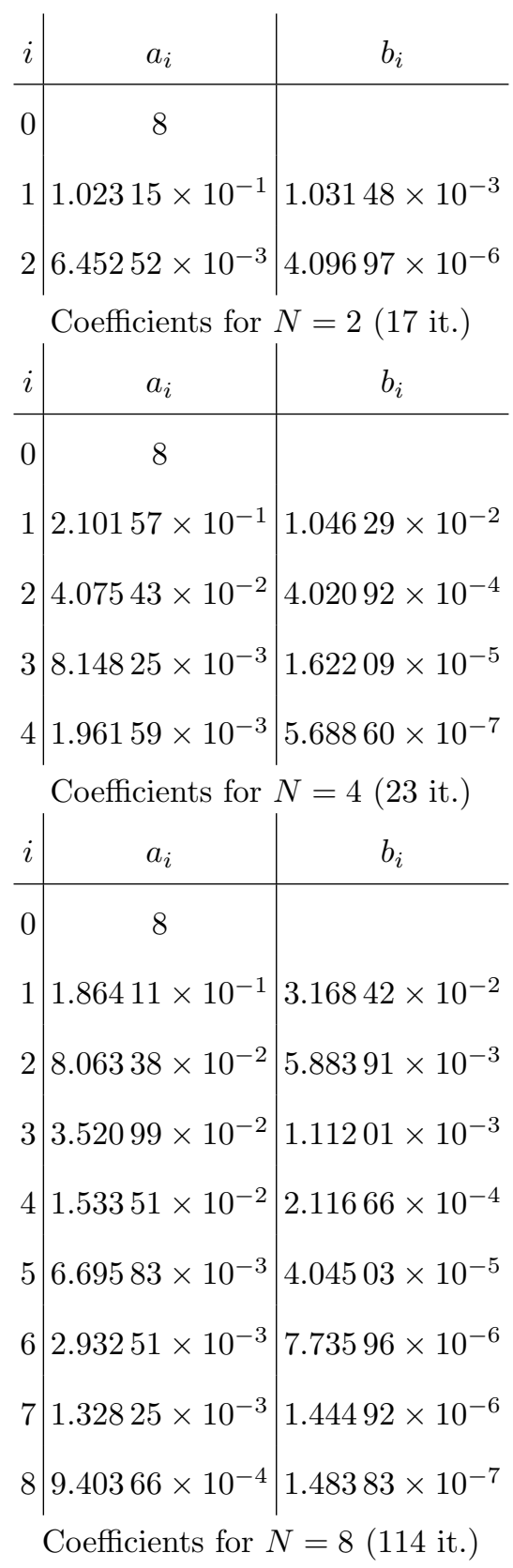

TABLE II. Coefficients $\left(a_{i}, b_{i}\right)$, optimized for $M=100$ values of $\zeta$ ranging from 8 to $2 \times 10^{6}$, covering radii of $1 \times 10^{-3} \mathrm{~m}$ to $0.1 \mathrm{~m}$ and frequencies of $20 \mathrm{~Hz}$ to $2 \times 10^{4} \mathrm{~Hz}$. In parentheses: number of iterations to reach the stagnation threshold $10^{-8}$. 
where the characteristic viscous and thermal times are given by

$$
\tau_{v}(x):=\frac{R(x)^{2} \rho}{\mu} \text { and } \tau_{\theta}(x):=\frac{R(x)^{2} \rho C_{P}}{\kappa}
$$

and the loss coefficients depend on

$$
G(\zeta):=\frac{j \zeta \phi(\sqrt{-j \zeta})}{1-\phi(\sqrt{-j \zeta})}, \quad \forall \zeta \in \mathbb{R}
$$

where $\phi$ is given by (2). The nondimensionalized frequency $\zeta$ in the viscous (resp. thermal) term will take values equal (resp. close to) the squared Stokes number $s^{2}$. Its range of interest, for applications in musical acoustics, lies approximately between $\zeta_{\min }=8$ (low frequency, thin pipes) and $\zeta_{\max }=2 \times 10^{6}$ (high frequency, wide pipes). This corresponds to frequencies of $20 \mathrm{~Hz}$ to $20 \mathrm{kHz}$ and radii of $1 \mathrm{~mm}$ to $100 \mathrm{~mm}$, subject to $\omega R<<c$ in order for model (1) to be valid. Function $G$ tends to the constant value 8 as $\zeta$ tends to zero, while for large $\zeta$, it is asymptotically equivalent to $2 \sqrt{j \zeta}$. Replacing $G$ by its asymptotic expansion as $\zeta \rightarrow \infty$ leads to fractional operators in time coming from the term $\sqrt{j \omega}$, which can be dealt with in the time domain using diffusive representations (Hélie and Matignon, 2006).

Instead, in the same spirit as (Bilbao and Harrison, 2016), function $G$ is approximated in the whole range of interest with a family of functions $G^{N}$ of the following form

$$
G^{N}(\zeta)=a_{0}+\sum_{i=1}^{N} \frac{a_{i} j \zeta}{b_{i} j \zeta+1}
$$




\section{Viscothermal time model for wind instruments}

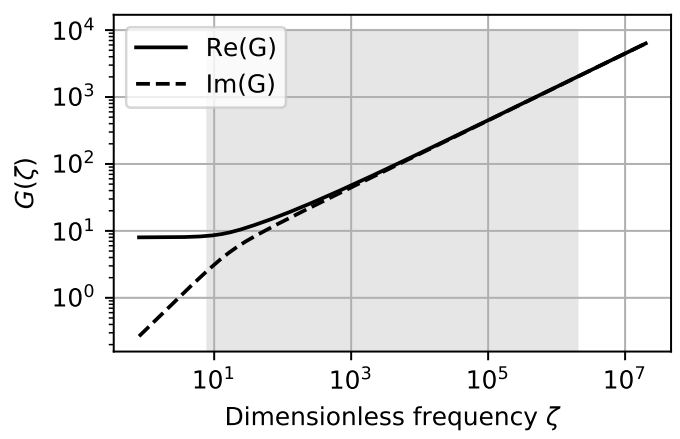

FIG. 1. Dimensionless loss coefficient $G(\zeta)$, w.r.t. dimensionless frequency $\zeta$. The range of interest $\left[\zeta_{\min }, \zeta_{\max }\right]$ is highlighted in gray.

\section{A. Optimization of the model coefficients}

The objective function is chosen to be

$$
E=\sum_{k=1}^{M}\left|\frac{G^{N}\left(\zeta_{k}\right)}{G\left(\zeta_{k}\right)}-1\right|^{2}
$$


Viscothermal time model for wind instruments

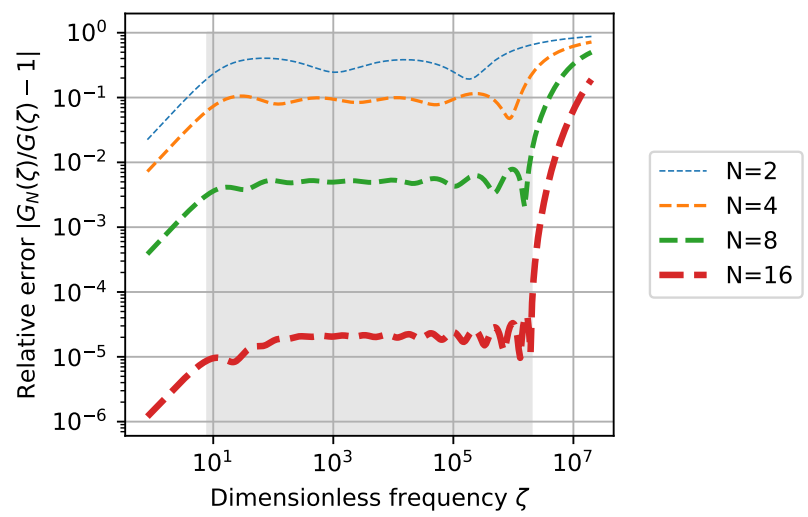

FIG. 2. Relative error between $G$ and $G^{N}$, as a function of dimensionless frequency $\zeta$, for several values of $N$. The range of interest $\left[\zeta_{\min }, \zeta_{\max }\right]$ is highlighted in gray.

where $\zeta_{k}$ are $M=100$ exponentially spaced values, spanning the range of interest $\left[\zeta_{\min }, \zeta_{\max }\right]$.

The positivity of $\left(a_{i}, b_{i}\right)_{i=1 \ldots N}$ is enforced by expressing them as $\left(\exp \left(a_{i}^{\prime}\right), \exp \left(b_{i}^{\prime}\right)\right)$. This reparametrization warrants the use of unconstrained optimization algorithms, and is suitable to control the model's behavior on frequencies spanning several orders of magnitude. The BFGS algorithm (Nocedal and Wright, 2006) is then used to find a minimizer of function $E\left(\left(a_{i}^{\prime}, b_{i}^{\prime}\right)_{i=1 \ldots N}\right)$. Table II gives values of coefficients $\left(a_{i}, b_{i}\right)$ that minimize this $\ell^{2}$ error, for different values of $N$. Note that these coefficients do not depend on the geometry of the instrument, and can be readily used as given. The choice of $N$ is a trade-off between the 
better precision enabled by using more additional variables, and the higher computing power required for simulation (see Sec. IV D).

\section{B. Resulting family of models in the frequency domain}

Replacing $G$ with $G^{N}$ yields the following family of approximate models:

$$
\left\{\begin{array}{l}
\frac{d \hat{p}}{d x}+\frac{\rho}{S}\left[j \omega+\frac{a_{0}}{\tau_{v}}+\sum_{i=1}^{N} \frac{a_{i} j \omega}{b_{i} \tau_{v} j \omega+1}\right] \hat{v}=0, \\
\frac{d \hat{v}}{d x}+\frac{S}{\rho c^{2}}\left[j \omega+\frac{\gamma-1}{\frac{1}{j \omega}+\frac{1}{\frac{a_{0}}{\tau_{\theta}}+\sum_{i=1}^{N} \frac{a_{i} j \omega}{b_{i} \tau_{\theta} j \omega+1}}}\right] \hat{p}=0,
\end{array}\right.
$$

These equations can be written and represented using an equivalent electronic circuit with Foster structure at each abscissa $x$, as is done in (Bilbao and Harrison, 2016), by defining the coefficients $R_{i}, L_{i}, C_{i}$ and $G_{i}$ as in equations (4). Such a representation is useful, not only to obtain an explicit choice of state-space representation for the rational functions in (13), but also for the derivation of the energy balance. 


\section{Resulting family of models in the time domain}

This family of models can be written in the time domain as follows

$$
\left(\mathcal{M}_{N}\right)\left\{\begin{array}{l}
\frac{\rho}{S} \frac{\partial v}{\partial t}+\frac{\partial p}{\partial x}+\Delta=0 \\
\frac{S}{\rho c^{2}} \frac{\partial p}{\partial t}+\frac{\partial v}{\partial x}+m=0 \\
\Delta=\Delta_{0}+\sum_{i=1}^{N} \Delta_{i}, \quad m=m_{0}+\sum_{i=1}^{N} m_{i} \\
\Delta_{0}=R_{0} v, \quad m_{0}=G_{0} q_{0}, \\
\Delta_{i}=R_{i} w_{i}=L_{i} d_{t} v_{i}, \quad v=w_{i}+v_{i} \\
m=C_{0} d_{t} p_{0}, \quad p=p_{0}+q_{0}, \\
m_{i}=C_{i} d_{t} p_{i}=G_{i} q_{i}, \quad q_{0}=p_{i}+q_{i} .
\end{array}\right.
$$

\section{Energy balance}

The energy identity comes from the circuit representation (Bilbao and Harrison, 2016), and the associated equations (14). Let us multiply the first equation with $v$ and the second equation with $p$, and integrate both in space over $[0, L]$. The terms $v \Delta$ and $p m$ that arise can be interpreted as the power brought into the electronic circuit. They can be written, 
using the rest of system (14), as

$$
\left\{\begin{array}{l}
v \Delta=R_{0} v^{2}+\sum_{i=1}^{N}\left(R_{i} w_{i}^{2}+\frac{L_{i}}{2} \frac{\mathrm{d} v_{i}^{2}}{\mathrm{~d} t}\right) \\
p m=\frac{C_{0}}{2} \frac{\mathrm{d} p_{0}^{2}}{\mathrm{~d} t}+G_{0} q_{0}^{2}+\sum_{i=1}^{N}\left(G_{i} q_{i}^{2}+\frac{C_{i}}{2} \frac{\mathrm{d} p_{i}^{2}}{\mathrm{~d} t}\right)
\end{array}\right.
$$

Eliminating the same unknowns as before establishes the energy balance identity (5) where the energy is defined as (6) and the losses are given by (7).

\section{PRACTICAL IMPLEMENTATION}

Space and time discretization of system (3) can be done with various numerical methods depending on the situation. In the context of sound synthesis, it is essential to design stable, accurate and efficient numerical schemes that couple the pipe with other elements such as the radiation at the bell, junctions with tone holes, or the embouchure, that can behave nonlinearly.

The current article proposes to use one-dimensional finite elements in space followed by an energy-consistent time discretization, in order to ensure numerical stability via an energy technique and to provide a numerical method that will easily extend to the presence of couplings. For the sake of simplicity, but without loss of generality, it is assumed that the outwards pipe flow is equal to given values $\lambda^{-}(t)$ and $\lambda^{+}(t)$ respectively at the pipe entrance 
and bell. In the presence of realistic coupling terms at the entrance and bell, these values will be unknowns and will require additional equations to be evaluated.

\section{A. One-dimensional finite elements for space discretization}

The finite element method (FEM) relies on a variational formulation (weak form) of the entire system in usual infinite dimensional functional spaces (Brezis, 2010) to which belong $p, v, p_{0}, p_{i}$ and $v_{i}$, followed by the definition of finite dimensional spaces in which we seek numerically the approximate solutions $p_{h}, v_{h}, p_{h, 0}, p_{h, i}$ and $v_{h, i}$. One possible choice, called Mixed Spectral FEM, is described in (Tournemenne and Chabassier, 2019) and is followed here. It consists in using as finite dimensional spaces the set of piecewise polynomial functions of the spatial variable $x$, element by element, where jumps across element edges are authorized for $v_{h}$ and $v_{h, i}$ but not for $p_{h}, p_{h, 0}$ and $p_{h, i}$. These polynomial functions are chosen as the Lagrange interpolation polynomials on the Gauss-Lobatto points of each element. The order of the polynomial functions is called the order of the FEM and will be noted $r$ thereafter. Finally, the integral terms coming from the variational formulation 
are evaluated using a quadrature formula based on the same Gauss-Lobatto points, so that mass matrices are diagonal (Cohen, 2013).

This procedure results in the definition of matrices that replace all the spatial operators in (3). The following "semi-discrete system" is obtained. Let $V_{h}, V_{h, i}, P_{h}, P_{h, 0}, P_{h, i}$ be the vectors of coordinates of resp. $v_{h}, v_{h, i}, p_{h}, p_{h, 0}$ and $p_{h, i}$ in the spanning basis of the finite elements, and see (Tournemenne and Chabassier, 2019) for the definition of the matrices $M_{h}^{V}, M_{h}^{P}, B_{h}, E_{h}$. The diagonal matrices $R_{h, 0}, R_{h, i}, G_{h, 0}, G_{h, i}, C_{h, 0}, C_{h, i}$ and $L_{h, i}$, are obtained via a similar procedure to the diagonal mass matrices $M_{h}^{V}$ and $M_{h}^{P}$.

$$
\left\{\begin{aligned}
M_{h}^{V} \frac{\partial V_{h}}{\partial t}+ & R_{h, 0} V_{h}+\sum_{i=1}^{N} R_{h, i}\left(V_{h}-V_{h, i}\right)-B_{h} P_{h}=0 \\
M_{h}^{P} \frac{\partial P_{h}}{\partial t}+ & G_{h, 0}\left(P_{h}-P_{h, 0}\right)+\sum_{i=1}^{N} G_{h, i}\left(P_{h}-P_{h, 0}-P_{h, i}\right) \\
& +B_{h}^{*} V_{h}+\lambda_{+} E_{h}^{+}+\lambda_{-} E_{h}^{-}=0 \\
L_{h, i} \frac{\partial V_{h, i}}{\partial t}= & R_{h, i}\left(V_{h}-V_{h, i}\right), \text { for } 1 \leq i \leq N \\
C_{h, 0} \frac{\partial P_{h, 0}}{\partial t}= & G_{h, 0}\left(P_{h}-P_{h, 0}\right) \\
& +\sum_{i=1}^{N} G_{h, i}\left(P_{h}-P_{h, 0}-P_{h, i}\right), \\
C_{h, i} \frac{\partial P_{h, i}}{\partial t}= & G_{h, i}\left(P_{h}-P_{h, 0}-P_{h, i}\right), \quad \text { for } 1 \leq i \leq N .
\end{aligned}\right.
$$

One advantage of this formulation is the natural treatment of the boundary conditions $\lambda_{ \pm}$, which can become Lagrange multipliers for coupling with other systems. This semi-discrete 
Viscothermal time model for wind instruments

system satisfies an analogue of the previous energy identity (5) where the continuous spatial norms are replaced with their semi discrete counterparts.

Note that the semi-discrete system (16) can be straightforwardly adapted for computation in the harmonic regime. This leads to one totally discrete system per value of $\omega$, where the operator $\partial_{t}$ is replaced with a multiplication with $j \omega$, which requires a sparse matrix inversion per $\omega$.

\section{B. Energy consistent time discretization}

For time discretization, an interleaved scheme similar to (Bilbao and Harrison, 2016) is used. The time step is denoted $\Delta t$, and the step number $n$, so that $t=n \Delta t$. Unknowns $P_{h}, P_{h, 0}$ and $P_{h, i}$ are evaluated at integer times $\{0,1,2, \ldots\}$, whereas $V_{h}$ and $V_{h, i}$ are evaluated on a staggered time grid $\{1 / 2,3 / 2,5 / 2, \ldots\}$. Let us define the discrete operators $\delta$ and $\mu$ acting on any vector sequence $\left\{X^{n}\right\}_{n \in \mathbb{N}}$ as

$$
\delta X^{n+\frac{1}{2}}=\frac{X^{n+1}-X^{n}}{\Delta t}, \mu X^{n+\frac{1}{2}}=\frac{X^{n+1}+X^{n}}{2} .
$$


Viscothermal time model for wind instruments

Conversely, if $\left\{Y^{n+\frac{1}{2}}\right\}_{n}$ is a sequence with indices on the staggered grid, $\left\{\delta Y^{n}\right\}_{n}$ is defined at integer times. The proposed totally discrete scheme reads

$$
\left\{\begin{array}{c}
M_{h}^{V} \delta V_{h}^{n}+R_{h, 0} \mu V_{h}^{n}+\sum_{i=1}^{N} R_{h, i} \mu\left(V_{h}-V_{h, i}\right)^{n} \\
-B_{h} P_{h}^{n}=0, \\
M_{h}^{P} \delta P_{h}^{n+\frac{1}{2}}+G_{h, 0} \mu\left(P_{h}-P_{h, 0}\right)^{n+\frac{1}{2}} \\
+\sum_{i=1}^{N} G_{h, i} \mu\left(P_{h}-P_{h, 0}-P_{h, i}\right)^{n+\frac{1}{2}}+B_{h}^{*} V_{h}^{n+\frac{1}{2}} \\
+\lambda_{+}^{n+\frac{1}{2}} E_{h}^{+}+\lambda_{-}^{n+\frac{1}{2}} E_{h}^{-}=0, \\
L_{h, i} \delta V_{h, i}^{n}=R_{h, i} \mu\left(V_{h}-V_{h, i}\right)^{n}, \forall i, \\
C_{h, 0} \delta P_{h, 0}^{n+\frac{1}{2}}=G_{h, 0} \mu\left(P_{h}-P_{h, 0}\right)^{n+\frac{1}{2}} \\
+\sum_{i=1}^{N} G_{h, i} \mu\left(P_{h}-P_{h, 0}-P_{h, i}\right)^{n+\frac{1}{2}}, \\
C_{h, i} \delta P_{h, i}^{n+\frac{1}{2}}=G_{h, i} \mu\left(P_{h}-P_{h, 0}-P_{h, i}\right)^{n+\frac{1}{2}}, \forall i .
\end{array}\right.
$$

where the discrete energy is defined as

$$
\mathcal{E}_{h}^{n}=\mathcal{E}_{h, V}^{n}+\mathcal{E}_{h, P}^{n}+\mu \mathcal{E}_{h, \mathrm{visc}}^{n}+\mathcal{E}_{h, \text { therm }}^{n}+\mathfrak{e}_{h}^{n}
$$


The different terms of energy are defined as

$$
\begin{gathered}
\mathcal{E}_{h, V}^{n}:=\frac{1}{2}\left\|\mu V_{h}^{n}\right\|_{M_{h}^{V}}^{2}, \quad \mathcal{E}_{h, P}^{n}:=\frac{1}{2}\left\|P_{h}^{n}\right\|_{\widetilde{M}_{h}^{P}}^{2} \\
\mathcal{E}_{h, \mathrm{visc}}^{n+\frac{1}{2}}:=\sum_{i=1}^{N} \frac{1}{2}\left\|V_{h, i}^{n+\frac{1}{2}}\right\|_{L_{h, i}}^{2} \\
\mathcal{E}_{h, \text { therm }}^{n}:=\frac{1}{2}\left\|P_{h, 0}^{n}\right\|_{C_{h, 0}}^{2}+\sum_{i=1}^{N} \frac{1}{2}\left\|P_{h, i}^{n}\right\|_{C_{h, i}}^{2} \\
\mathfrak{e}_{h}^{n}:=\frac{\Delta t^{2}}{8}\left\|\mu V_{h}^{n}+\sum_{i=1}^{N} R_{h, 0}^{-1} R_{h, i} \mu\left(V_{h}-V_{h, i}\right)^{n}\right\|_{\widetilde{R}_{h, 0}}^{2} \\
Q_{h, \mathrm{visc}}^{n}:=\left\|\mu V_{h, 0}^{n}\right\|_{R_{h, 0}}^{2}+\sum_{i=1}^{N}\left\|\mu\left(V_{h}-V_{h, i}\right)^{n}\right\|_{R_{h, i}}^{2} \\
Q_{h, \mathrm{therm}}^{n+\frac{1}{2}}:=\left\|\mu\left(P_{h}-P_{h, 0}\right)^{n+\frac{1}{2}}\right\|_{G_{h, 0}}^{2} \\
\quad+\sum_{i=1}^{N}\left\|\mu\left(P_{h}-P_{h, 0}-P_{h, i}\right)^{n+\frac{1}{2}}\right\|_{G_{h, i}}^{2} \\
\mathcal{S}_{h}^{n+\frac{1}{2}}:=-\lambda_{+}^{n+\frac{1}{2}}\left(E_{h}^{+}\right)^{*} \mu P_{h}^{n+\frac{1}{2}}-\lambda_{-}^{n+\frac{1}{2}}\left(E_{h}^{-}\right)^{*} \mu P_{h}^{n+\frac{1}{2}}
\end{gathered}
$$

where

$$
\widetilde{R}_{h, 0}=R_{h, 0}\left(M_{h}^{V}\right)^{-1} R_{h, 0}
$$

and for any time series of vectors $\left\{X^{n}\right\}_{n},\left\{Y^{n}\right\}_{n}$, their scalar product is defined as $\left(X^{n}, Y^{m}\right)=\sum_{k} X_{k}^{n} Y_{k}^{m}$ and the weighted norm, for any non negative matrix $\mathrm{A}$, is $\left\|X^{n}\right\|_{A}=$ $\left(A X^{n}, X^{n}\right)^{1 / 2}$. This energy is positive as soon as the modified mass matrix $\widetilde{M}_{h}^{P}$, defined as $\widetilde{M}_{h}^{P}=M_{h}^{P}-\frac{\Delta t^{2}}{4} B_{h}^{*}\left(M_{h}^{V}\right)^{-1} B_{h}$ is a positive quadratic form. This leads to the following stability condition:

$$
\Delta t \leq 2\left[\rho\left(\left(M_{h}^{P}\right)^{-1} B_{h}^{*}\left(M_{h}^{V}\right)^{-1} B_{h}\right)\right]^{-\frac{1}{2}}
$$


Viscothermal time model for wind instruments

which is the same condition as for solving the lossless wave equation with the classical leapfrog scheme (Chabassier and Imperiale, 2013). Showing the convergence of the discrete scheme, as $\Delta t$ and $h$ tend to zero, is out of the scope of the present work but it is expected that the dissipation of a positive energy will enable such a result (Chabassier and Imperiale, 2017).

\section{Explicit update of the unknowns}

One of the advantages of the of spectral finite elements is that $M_{h}^{P}, M_{h}^{V}, L_{h, i}, C_{h, 0}$ and $C_{h, i}$ are diagonal matrices, making them trivial to invert. Thanks to an algorithmic elimination strategy, which can also be interpreted as a Schur complement, an explicit update of the unknowns is possible and is given in Appendix B. This approach generalizes the one given in Table I of (Bilbao and Harrison, 2016) for finite differences, to finite elements of arbitrary order in matrix notations. 


\section{Accuracy and efficiency}

Model (18) is applied to a simplified natural trumpet of total length $1.335 \mathrm{~m}$. The pipe comprises two parts: a cylinder, followed by a "Bessel horn":

$$
R(x)= \begin{cases}6 \times 10^{-3} \mathrm{~m} & \text { if } 0<x<0.716, \\ \gamma\left(x-x_{P}\right)^{-\alpha} & \text { if } 0.716<x<1.335\end{cases}
$$

where $\alpha=0.7$ is the parameter of the Bessel horn. Constants $\gamma=4.40437 \times 10^{-3} \mathrm{~m}$ and $x_{P}=1.35897 \mathrm{~m}$ are chosen such that the radius is continuous at the junction, and that the bell radius is $60 \mathrm{~mm}$, see Fig. 3. For simplicity, we assume a zero-pressure condition at the bell.

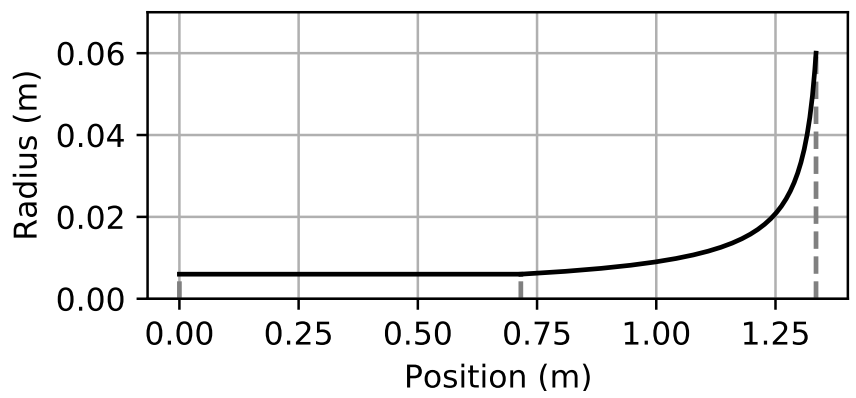

FIG. 3. The simplified natural trumpet : radius w.r.t. position along bore 
An impulse response is computed by setting the input boundary term to a prescribed flow $-\lambda_{-}^{n+\frac{1}{2}}=v_{0}\left(\left(n+\frac{1}{2}\right) \Delta t\right)$, with

$$
v_{0}(t)= \begin{cases}\frac{8 V_{0}}{3 t_{1}} \sin ^{4}\left(\pi \frac{t}{t_{1}}\right) & \text { if } 0<t<t_{1} \\ 0 & \text { otherwise }\end{cases}
$$

where the duration of the impulse is $t_{1}=4 \times 10^{-4} \mathrm{~s}$ and the total injected volume is $V_{0}=1 \times 10^{-7} \mathrm{~m}^{3}$. At the bell an elementary open condition $p=0$ is applied: the generalization to more realistic radiation impedances is a natural possible extension of the present work.

The numerical scheme (18) is used to compute the impulse response at the temperature $t=20^{\circ} \mathrm{C}$ with 34 elements of order 10 and a time step $\Delta t=3.185 \times 10^{-6} \mathrm{~s}$, which is the largest value satisfying the stability condition (21). The final time is set to $T=0.2 \mathrm{~s}$.

Fig. 4 displays the computed impulse response, i.e. the evolution of the pressure at the entrance of the simplified trumpet with respect to time, for $N \in\{2,4,8,16\}$, between 0 s and $0.2 \mathrm{~s}$ (top), $0.13 \mathrm{~s}$ and $0.18 \mathrm{~s}$ (bottom). The main reflections from the pipe ends can be observed, as well as partial reflections coming from the radius variation in the Bessel part of the pipe. Despite the lack of dissipation at the pipe ends, the impulse response is decaying 

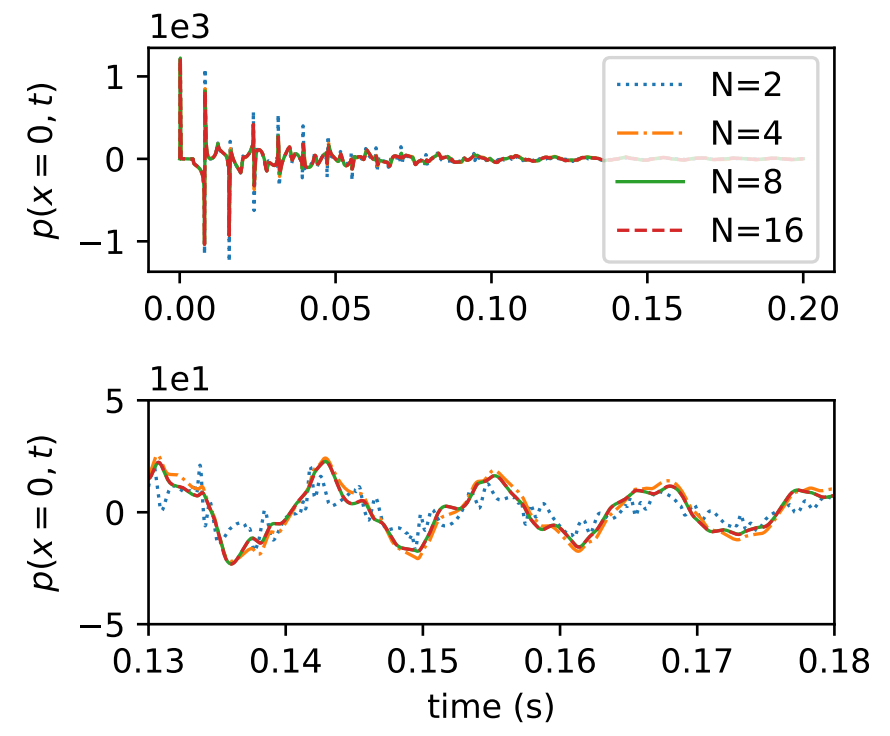

FIG. 4. Impulse response: evolution of the pressure at the entrance of the simplified trumpet with respect to time, for $N \in\{2,4,8,16\}$, between $0 \mathrm{~s}$ and $0.2 \mathrm{~s}$ (top), $0.13 \mathrm{~s}$ and $0.18 \mathrm{~s}$ (bottom). The curves for $N=8$ and $N=16$ cannot be visually distinguished.

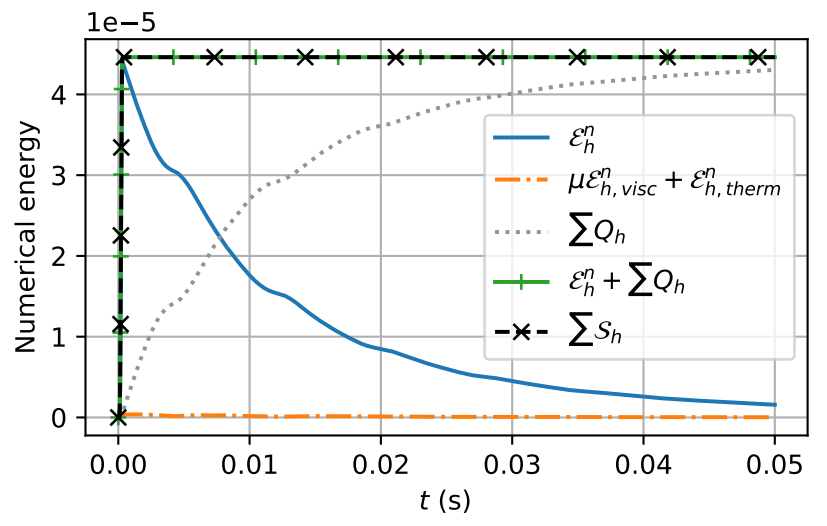

FIG. 5. Energy distribution w.r.t. time 
Fig 5 displays the energy distribution with respect to time, according to the definitions given in Sec. IV B. The total energy in the pipe, brought by the impulse input flow, is globally decaying after $t_{1}$, and is temporarily stored in the auxiliary variables through the viscous and thermal energy terms. The energy identity (19) is satisfied up to machine precision as illustrated in Fig. 6.

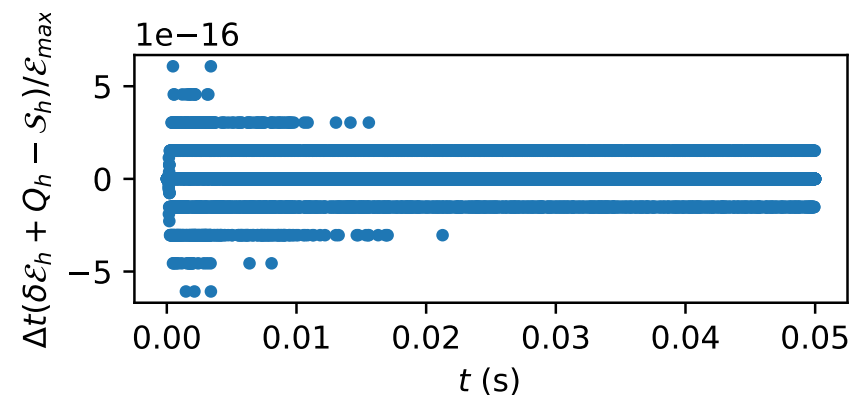

FIG. 6. Energy relative deviation w.r.t. time

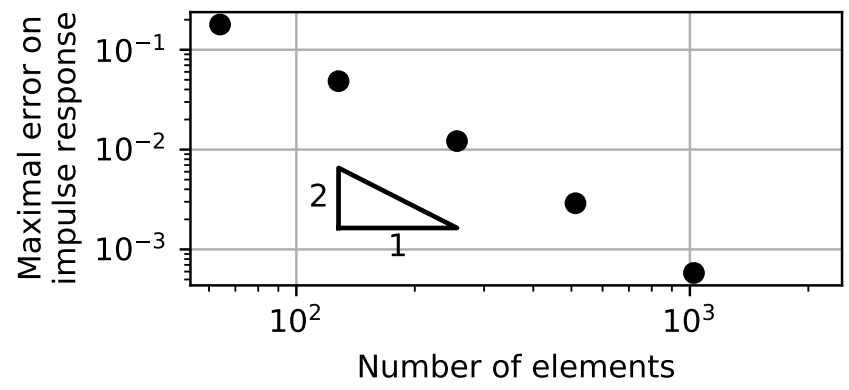

FIG. 7. Consecutive relative $L^{\infty}([0, T], x=0)$ error w.r.t. the number of finite elements of order 10. 
Finally Fig. 7 shows the consecutive relative $L^{\infty}$-error in time of the impulse response with respect to the number of finite elements. As the spatial discretization of the pipe is refined, the time step is decreased by choosing the largest possible value given by the stability condition (21). The obtained impulse response is interpolated on a fixed time grid and compared. The resulting space-time convergence curve displays a second order rate of convergence.

\section{COMPARISON WITH OTHER MODELS}

Model (3) is compared with several models of the literature, in time domain and in frequency domain. The comparison includes two reduced 1D models: model (ZK) (1) and Webster-Loskhin model (WL) developed in (Hélie et al., 2013), without the hypothesis of curved wavefronts, which is out of the scope of the present article. They are compared to 3D linear acoustic equations in the air column, associated with effective wall impedance boundary conditions of two types: from (Cremer, 1948) with an incident angle of $\pi / 2$ noted (Cr), and from (Berggren et al., 2018) noted (BBN). All 3D solutions are obtained using finite elements for the spatial discretization using the software Montjoie $^{2}$, in an axisymmetric configuration. The numerical solution relies on a curved mesh of the simplified natural 
trumpet. The input boundary condition is a constant pressure set at the extremity of an cylindrical adaptation part, and the output boundary condition is a zero acoustic pressure on a plane surface which closes the pipe. The reader is referred to (Thibault and Chabassier, 2020) for a discussion of the domain of validity of each model, a detailed description of the 3D simulations (Sec. 3) and the implementation of model (WL) (Sec. 4.4). All 1D solutions are obtained using the software OpenWiND ${ }^{3}$.
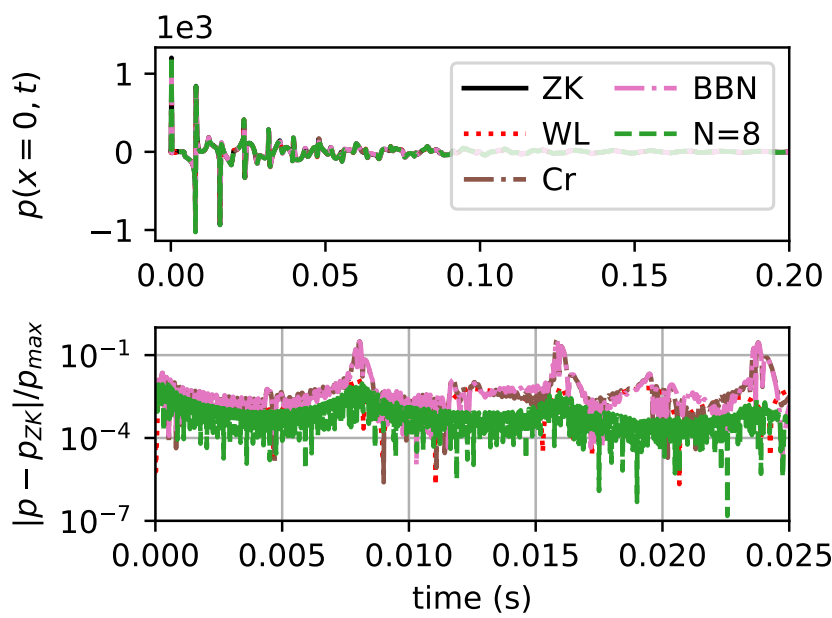

FIG. 8. Impulse response: evolution of the pressure at the entrance of the simplified trumpet with respect to time, for different models : ZK, WL, scheme (18) with $\mathrm{N}=8$, Cr and BBN, between $0 \mathrm{~s}$ and $0.2 \mathrm{~s}$ (top). Relative difference of each impulse response with ZK between $0 \mathrm{~s}$ and $0.025 \mathrm{~s}$ (bottom).

A first comparison concerns the impulse response obtained with each model. Models $(\mathrm{ZK}),(\mathrm{WL}),(\mathrm{Cr})$ and $(\mathrm{BBN})$ are all formulated in the frequency domain, and are suited 
to calculating an input impedance $Z_{i n}(\omega)$ where $\omega=2 \pi f$ is the dual variable to the time variable $t$. The response is calculated by taking the fast Fourier transform of the input signal (22), multiplying at each frequency by the impedance, and applying the inverse fast Fourier transform to the result. The sampling frequency is chosen to be $52747.2 \mathrm{~Hz}$, so that the error on the input signal due to spectral folding is less than $1 \times 10^{-5}$. The computation is done with $n=52747$ samples $(1.0 \mathrm{~s})$, and requires to evaluate the impedance of each model at 26373 frequencies (negative frequencies are obtained by conjugate symmetry). The resulting signals are displayed in Fig. 8. They decay below $2 \times 10^{-6}$ of their maximal value after $0.9 \mathrm{~s}$, meaning the error due to periodization is negligible.

A second comparison is done on the input impedance. System (16) is solved, where the operator $\partial_{t}$ has been replaced with $j \omega$, for $\omega \in\left\{\omega_{j}\right\}_{1 \leq j \leq M}$, $M$ linearly spaced pulsations in the range $\left[\omega^{-}, \omega^{+}\right]$. Model (3) is first solved with $N=2,4,8,16$ and compared with the other models. Fig 9 displays a focus around the second impedance peak of the simplified natural trumpet. As $N$ increases, the second impedance peak shifts in frequency and in quality factor, and reaches values that can not be visually distinguished from model (ZK) for $N=8$ and $N=16$. Models $(\mathrm{Cr})$ and $(\mathrm{BBN})$ are very close to the $1 \mathrm{D}$ models, $(\mathrm{Cr})$ being 


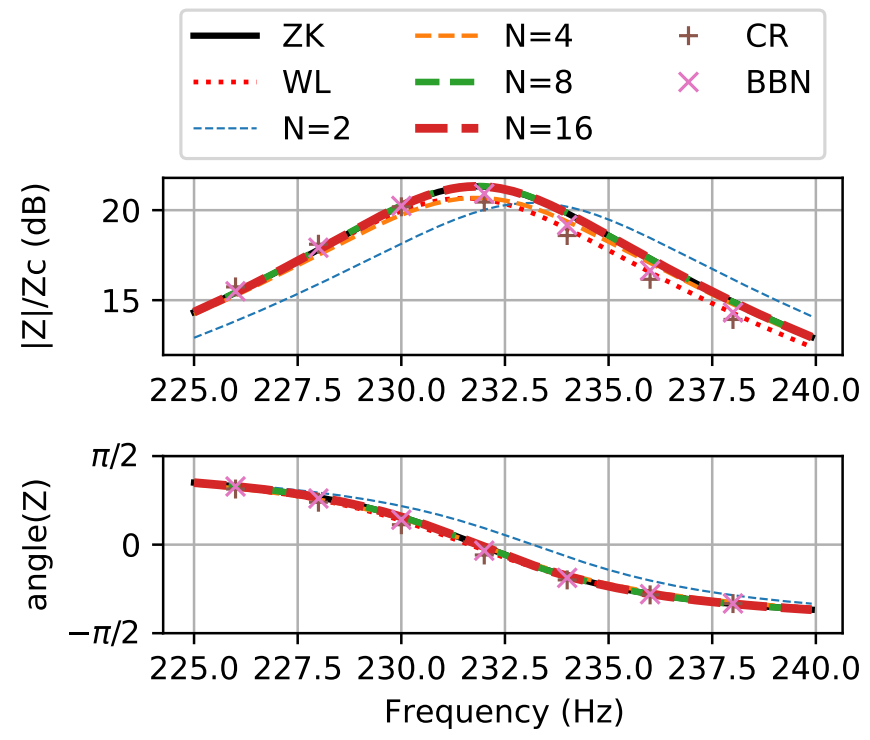

FIG. 9. Amplitude (top) and angle (bottom) of the input impedance computed of the simplified natural trumpet with model (3) for $N=2,4,8,16$ (dashed lines of increasing width), compared with modes (ZK) (thick black line), (Cr) (plus marker) and (BBN) (x marker). Focus around the second impedance peak.

closer to (WL) which indeed relies on Cremer wall impedances, and (BBN) to (ZK). It can be noted that the difference in resonance frequency (up to $1 \%$ i.e. 17 cents) between models is of the same order as the differential pitch sensitivity of the ear (around 4 cents) (Micheyl et al., 2006).

Fig. 10 quantitatively compares the models. With model (ZK) taken as a reference, the largest error of the other models on the impulse response is calculated. This error amounts to $1.5 \%$ for (WL). For the numerical schemes, the discrepancy results from two kinds of 


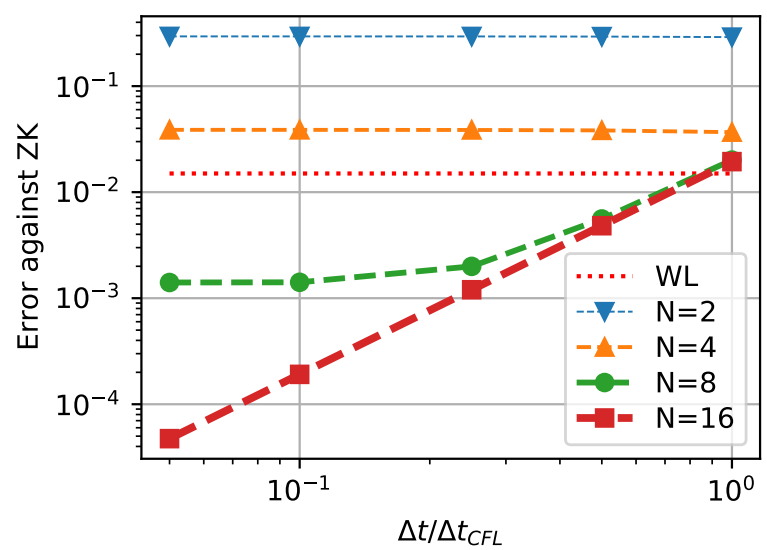

FIG. 10. Relative $L^{\infty}$ error on the impulse response computed with $N \in 2,4,8,16$ (markers) and reconstructed from WL (no marker) with respect to ZK model.

error: model error, and discretization error. The former is due to approximation (11) and can be reduced by increasing $N$; the latter is due to the numerical scheme (18) and can be reduced by decreasing the time step $\Delta t$, as done in Fig 10. For $N=8$ the model error compared to $(\mathrm{ZK})$ is observed to be about $0.14 \%$, and for $N=16$ it is less than $0.02 \%$.

\section{CONCLUSIONS AND PROSPECTS}

This work presents a 1D model for viscothermal wave propagation suitable for timedomain simulation, which makes use of an adjustable number of auxiliary unknowns. It is derived from an approximation of the loss coefficients of the Zwikker-Kosten model with rational functions over the whole range of frequencies and radii of musical acoustics. The 
Viscothermal time model for wind instruments

317

proposed improvement upon previous work is that the coefficient optimization procedure has been done only once, as the objective function does not depend on temperature or pipe radius. It leads to a closed-form for the resulting model, where the user only needs to specify the instrument geometry and physical constants. Numerical comparison with 3D models show that the approximation error is smaller than the discrepancies between different models. The use of auxiliary variables induces a numerical burden which was to be expected for accounting for viscothermal effects. The model satisfies an energy identity and is therefore suitable for time-domain coupling with other models as sound radiation, or reed evolution, which are a possible extension of this work. This work could be extended to include additional forms of acoustic losses, such as wall admittance due to porosity, or to better justify its use in tubes with variable cross-sectional area. 


\section{APPENDIX A: DISCRETE ENERGY BALANCE}

\section{Useful identities}

Using discrete-time operators $\mu$ and $\delta$ defined in (17), the following identities, implicitly centered in $t^{n+\frac{1}{2}}$, hold

$$
\left\{\begin{array}{l}
(\mu X, \mu Y)+\frac{\Delta t^{2}}{4}(\delta X, \delta Y)=\mu(X, Y), \\
\mu \mu X=X+\frac{\Delta t^{2}}{4} \delta \delta X \\
(\mu X, \delta X)=\delta\left(\frac{1}{2}\|X\|^{2}\right) .
\end{array}\right.
$$

\section{Notations}

In this appendix, for the sake of brevity, subscripts $h$ and time integers $n$ and $n+1 / 2$ are omitted. We denote

$$
\begin{array}{ll}
\Delta_{0}=R_{0} \mu V, & m_{0}=G_{0} \mu\left(P-P_{0}\right), \\
\Delta_{i}=R_{i} \mu\left(V-V_{i}\right), & m_{i}=G_{i} \mu\left(P-P_{0}-P_{i}\right), \\
\Delta=\Delta_{0}+\sum_{i} \Delta_{i}, & m=m_{0}+\sum_{i} m_{i} .
\end{array}
$$

331 Notice that $\Delta_{i} \stackrel{(18 \mathrm{c})}{\equiv} L_{i}, \delta V_{i}, m_{i} \stackrel{(18 \mathrm{e})}{\equiv} C_{i} \delta P_{i}, m \stackrel{(18 \mathrm{~d})}{\equiv} C_{0} \delta P_{0}$. Moreover we use the notations 332 defined in section IV B. 


\section{Energy derivation}

Taking the scalar product of $\mu(18 \mathrm{a})$ with $\mu \mu V$ yields $\left(M^{V} \mu \delta V+\mu \Delta, \mu \mu V\right)-(B \mu P, \mu \mu V)=$

0. The scalar product of $(18 \mathrm{~b})$ with $\mu P$ gives

$$
\left(M^{P} \delta P+m, \mu P\right)+\left(B^{*} V, \mu P\right)
$$

$$
+\underbrace{\left(\lambda_{-} E^{-}+\lambda_{+} E^{+}, \mu P\right)}_{-\mathcal{S}_{h}^{n+\frac{1}{2}}}=0 .
$$

Then sum the two previous results and use identity (A1c) twice

$$
\begin{aligned}
\delta \frac{1}{2}\|\mu V\|_{M^{V}}^{2}+\delta \frac{1}{2}\|P\|_{M^{P}}^{2}+\overbrace{(\mu \Delta, \mu \mu V)}^{\text {A }}+\overbrace{(m, \mu P)}^{\text {B }} & +\underbrace{\left(\mu P, B^{*}(V-\mu \mu V)\right)}_{\text {C }}=\mathcal{S}_{h}^{n+\frac{1}{2}} .
\end{aligned}
$$

Terms (A), B and (C) are treated separately.

$$
\text { A } \stackrel{(\mathrm{A} 1 \mathrm{a})}{=} \mu(\Delta, \mu V)-\frac{\Delta t^{2}}{4}(\delta \Delta, \delta \mu V) .
$$

Now, the discrete work $(\Delta, \mu V)$ writes

$$
\begin{aligned}
(\Delta, \mu V)=\left(\mu V, \Delta_{0}+\sum_{i} \Delta_{i}\right) & \\
=\left(\mu V, R_{0} \mu V\right) & +\sum_{i}(\mu V_{i}, \overbrace{\Delta_{i}}^{L_{i} \delta V}) \\
& +\sum_{i}(\mu\left(V-V_{i}\right), \underbrace{\Delta_{i}}_{R_{i} \mu\left(V-V_{i}\right)}) \\
& =\|\mu V\|_{R_{0}}^{2}+\sum_{i}\left[\delta \frac{1}{2}\left\|V_{i}\right\|_{L_{i}}^{2}+\left\|\mu\left(V-V_{i}\right)\right\|_{R_{i}}^{2}\right]
\end{aligned}
$$


using (A1c) again. Hence

$$
\begin{aligned}
\mathrm{A}=\mu\|\mu V\|_{R_{0}}^{2}-\frac{\Delta t^{2}}{4}(\delta \Delta, \delta \mu V) & \\
& +\mu \sum_{i}\left[\delta \frac{1}{2}\left\|V_{i}\right\|_{L_{i}}^{2}+\left\|\mu\left(V-V_{i}\right)\right\|_{R_{i}}^{2}\right]
\end{aligned}
$$

Moreover, the discrete work $(m, \mu P)$ writes

$$
\begin{aligned}
& \text { (B) }=(\underbrace{m}_{C_{0} \delta P_{0}}, \mu P_{0})+(\underbrace{m}_{m_{0}+\sum_{i} m_{i}}, \mu\left(P-P_{0}\right)) \\
& =\delta \frac{1}{2}\left\|P_{0}\right\|_{C_{0}}^{2}+(\underbrace{m_{0}}_{G_{0} \mu\left(P-P_{0}\right)}, \mu\left(P-P_{0}\right)) \\
& \quad+\sum_{i}\left[(\underbrace{m_{i}}_{G_{i} \mu\left(P-P_{0}-P_{i}\right)}, \mu\left(P-P_{0}-P_{i}\right))+(\underbrace{m_{i}}_{C_{i} \delta P_{i}}, \mu P_{i})\right] \\
& =\delta \frac{1}{2}\left[\left\|P_{0}\right\|_{C_{0}}^{2}+\sum_{i}\left\|P_{i}\right\|_{C_{i}}^{2}\right]+\left\|\mu\left(P-P_{0}\right)\right\|_{G_{0}}^{2} \\
& \quad+\sum_{i}\left\|\mu\left(P-P_{0}-P_{i}\right)\right\|_{G_{i}}^{2}
\end{aligned}
$$

Finally, using (A1b) and commutativity of $\mu$ and $\delta$,

$$
\begin{aligned}
& \mathrm{C}=-\frac{\Delta t^{2}}{4}(\mu B P, \delta \delta V) \\
& \stackrel{(18 \mathrm{a})}{=}-\frac{\Delta t^{2}}{4}\left(\mu B P,\left(M^{V}\right)^{-1} \delta[B P-\Delta]\right) \\
& =\frac{\Delta t^{2}}{4}\left[-\delta \frac{1}{2}\|P\|_{B^{*}\left(M^{V}\right)^{-1} B}^{2}+(\mu \underbrace{B P}_{M^{V} \delta V+\Delta},\left(M^{V}\right)^{-1} \delta \Delta)\right] \\
& =\frac{\Delta t^{2}}{4}\left[-\delta \frac{1}{2}\|P\|_{B^{*}\left(M^{V}\right)^{-1} B}^{2}+(\delta \mu V, \delta \Delta)\right. \\
& \left.\quad+\delta \frac{1}{2}\|\Delta\|_{\left(M^{V}\right)^{-1}}^{2}\right]
\end{aligned}
$$




\section{APPENDIX B: EXPLICIT UPDATE OF THE UNKNOWNS}

Assume $P_{h}^{n}, P_{h, 0}^{n}, P_{h, i}^{n}, V_{h}^{n+1 / 2}, V_{h, i}^{n+1 / 2}$ are known. Define

$$
\begin{aligned}
\widetilde{G}_{h} & =G_{h, 0}+\sum_{i} G_{h, i}, \widetilde{R}_{h}=R_{h, 0}+\sum_{i} R_{h, i}, \\
\widetilde{C}_{h, 0} & =C_{h, 0}+\frac{\Delta t}{2} \widetilde{G}_{h}
\end{aligned}
$$

The next iterates $P_{h}^{n+1}, P_{h, 0}^{n+1}, P_{h, i}^{n+1}, V_{h}^{n+3 / 2}, V_{h, i}^{n+3 / 2}$ can be computed as follows, where all the matrices to invert are diagonal, beginning with $P_{h}^{n+1}$ :

$$
\begin{aligned}
& {\left[M_{h}^{P}+\frac{\Delta t}{2} \widetilde{C}_{h, 0}^{-1} C_{h, 0} \widetilde{G}_{h}\right] P_{h}^{n+1} }= \\
& {\left[M_{h}^{P}-\frac{\Delta t}{2} \widetilde{C}_{h, 0}^{-1} C_{h, 0} \widetilde{G}_{h}\right] P_{h}^{n}+\Delta t \widetilde{C}_{h, 0}^{-1} C_{h, 0} \widetilde{G}_{h} P_{h, 0}^{n} } \\
&+\Delta t \sum_{i} \widetilde{C}_{h, 0}^{-1}\left(G_{h, i}^{-1}+\frac{\Delta t}{2} C_{h, i}^{-1}\right)^{-1} C_{h, 0} P_{h, i}^{n} \\
& \quad-\Delta t\left[B_{h}^{*} V^{n+\frac{1}{2}}+\lambda_{+}^{n+\frac{1}{2}} E_{h}^{+}+\lambda_{-}^{n+\frac{1}{2}} E_{h}^{-}\right] .
\end{aligned}
$$

Now that $P_{h}^{n+1}$ is known, we compute

$$
\begin{aligned}
{\left[C_{h, 0}+\frac{\Delta t}{2} \widetilde{G}_{h}\right] P_{h, 0}^{n+1}=\left[C_{h, 0}-\frac{\Delta t}{2} \widetilde{G}_{h}\right] } & P_{h, 0}^{n} \\
& +\frac{\Delta t}{2} \widetilde{G}_{h}\left(P_{h}^{n}+P_{h}^{n+1}\right)-\Delta t \sum_{i}\left(G_{h, i}^{-1}+\frac{\Delta t}{2} C_{h, i}^{-1}\right)^{-1} P_{h, i}^{n} .
\end{aligned}
$$

Now that $P_{h, 0}^{n+1}$ is known, we compute

$$
\left[C_{h, i}+\frac{\Delta t}{2} G_{h, i}\right] P_{h, i}^{n+1}=\left[C_{h, i}-\frac{\Delta t}{2} G_{h, i}\right] P_{h, i}^{n}
$$$$
+\frac{\Delta t}{2} G_{h, i}\left(P_{h}^{n}+P_{h}^{n+1}-P_{h, 0}^{n}-P_{h, 0}^{n+1}\right) .
$$ 
Viscothermal time model for wind instruments

Since $P_{h}^{n+1}$ is known, we compute

$$
\left[M_{h}^{V}+\frac{\Delta t}{2} \widetilde{R}_{h}\right] V_{h}^{n+\frac{3}{2}}=\left[M_{h}^{V}-\frac{\Delta t}{2} \widetilde{R}_{h}\right] V_{h}^{n+\frac{1}{2}}
$$$$
+\Delta t \sum_{i}\left(R_{h, i}^{-1}+\frac{\Delta t}{2} L_{h, i}^{-1}\right)^{-1} V_{h, i}^{n+\frac{1}{2}}+\Delta t B P_{h}^{n+1}
$$

Finally, $V_{h, i}^{n+\frac{3}{2}}$ is obtained as

$$
\left[L_{h, i}+\frac{\Delta t}{2} R_{h, i}\right] V_{h, i}^{n+\frac{3}{2}}=\left[L_{h, i}-\frac{\Delta t}{2} R_{h, i}\right] V_{h, i}^{n+\frac{1}{2}}
$$

$$
+\frac{\Delta t}{2} R_{h, i}\left(V_{h}^{n+\frac{1}{2}}+V_{h}^{n+\frac{3}{2}}\right)
$$

${ }^{1}$ See supplementary material at https://asa.scitation.org/doi/suppl/10.1121/10.0005537 for the tables of coefficients $\left(a_{i}, b_{i}\right)$ for $N=0$ to 16 .

${ }^{2}$ http://montjoie.gforge.inria.fr

${ }^{3}$ http://openwind.gitlabpages.inria.fr/web

Abel, J., Smyth, T., and O. Smith, J. (2003). "A simple, accurate wall loss filter for acoustic tubes," International Conference on Digital Audio Effects 2003 Proceedings, London, UK 2003, 53-57. 
Berggren, M., Bernland, A., and Noreland, D. (2018). "Acoustic boundary layers as boundary conditions," Journal of Computational Physics 371, 633 - 650.

Berjamin, H., Lombard, B., Vergez, C., and Cottanceau, E. (2017). "Time-domain numerical modeling of brass instruments including nonlinear wave propagation, viscothermal losses, and lips vibration," Acta Acustica united with Acustica 103(1), 117-131.

Bilbao, S. (2009). "Direct simulation of reed wind instruments," Computer Music Journal $33(4), 43-55$.

Bilbao, S., Harrison, R., Kergomard, J., Lombard, B., and Vergez, C. (2015). "Passive models of viscothermal wave propagation in acoustic tubes," The Journal of the Acoustical Society of America 138(2), 555-558.

Bilbao, S., and Harrison, R. (2016). "Passive time-domain numerical models of viscothermal wave propagation in acoustic tubes of variable cross section," The Journal of the Acoustical Society of America 140(1), 728-740.

Brezis, H. (2010). Functional analysis, Sobolev spaces and partial differential equations (Springer Science \& Business Media).

Bruneau, M., Herzog, P., Kergomard, J., and Polack, J. (1989). "General formulation of the dispersion equation in bounded visco-thermal fluid, and application to some simple 
geometries," Wave motion 11(5), 441-451.

Chabassier, J., Diaz, J., and Imperiale, S. (2020). "Construction and analysis of fourth order, energy consistent, family of explicit time discretizations for dissipative linear wave equations," ESAIM: Mathematical Modelling and Numerical Analysis 54(3), 845-878.

Chabassier, J., and Imperiale, S. (2013). "Introduction and study of fourth order theta schemes for linear wave equations," Journal of Computational and Applied Mathematics 245, 194-212.

Chabassier, J., and Imperiale, S. (2017). "Space/time convergence analysis of a class of conservative schemes for linear wave equations," Comptes Rendus Mathematique 355(3), 282-289.

Chaigne, A., and Kergomard, J. (2016). Acoustics of musical instruments (Springer).

Chatziioannou, V., Schmutzhard, S., Pàmies-Vilà, M., and Hofmann, A. (2019). "Investigating clarinet articulation using a physical model and an artificial blowing machine," Acta Acustica United with Acustica 105(4), 682-694.

Cohen, G. (2013). Higher-order numerical methods for transient wave equations (Springer Science \& Business Media). 
Cremer, L. (1948). "On the acoustic boundary layer outside a rigid wall," Arch. Elektr. Uebertr 2, 235 .

Dalmont, J.-P., Nederveen, C. J., and Joly, N. (2001). "Radiation impedance of tubes with different flanges: numerical and experimental investigations," Journal of sound and vibration 244(3), 505-534.

Hélie, T., Hézard, T., Mignot, R., and Matignon, D. (2013). "One-dimensional acoustic models of horns and comparison with measurements," Acta acustica united with Acustica 99(6), 960-974.

Hélie, T., and Matignon, D. (2006). "Diffusive representations for the analysis and simulation of flared acoustic pipes with visco-thermal losses," Mathematical Models and Methods in Applied Sciences 16(4), 503-536.

Hélie, T., and Silva, F. (2017). "Self-oscillations of a vocal apparatus: a port-hamiltonian formulation," in International Conference on Geometric Science of Information, Springer, pp. $375-383$.

Jith, J., and Sarkar, S. (2018). "Boundary layer impedance model to analyse the viscothermal acousto-elastic interactions in centrifugal compressors," Journal of Fluids and Structures 81, 179-200. 
Kampinga, W., Wijnant, Y. H., and de Boer, A. (2011). "An efficient finite element model for viscothermal acoustics," Acta Acustica united with Acustica 97(4), 618-631.

Keefe, D. H. (1984). "Acoustical wave propagation in cylindrical ducts: Transmission line parameter approximations for isothermal and nonisothermal boundary conditions," The Journal of the Acoustical Society of America 75(1), 58-62.

Kirchhoff, G. (1868). "Ueber den einfluss der wärmeleitung in einem gase auf die schallbewegung," Annalen der Physik 210(6), 177-193.

Micheyl, C.,Delhommeau, K., Perrot X., , and Oxenham, A. J. (2006). "Influence of musical and psychoacoustical training on pitch discrimination," Hearing research 219(1-2), 36-47.

Mignot, R., Hélie, T., and Matignon, D. (2010). "Digital Waveguide Modeling for Wind Instruments: Building a State-Space Representation Based on the Webster-Lokshin Model," IEEE transactions on audio, speech, and language processing 18(4), 843-854.

Monteghetti, F., Matignon, D., and Piot, E. (2018). "Energy analysis and discretization of nonlinear impedance boundary conditions for the time-domain linearized euler equations," Journal of Computational Physics 375, 393-426. 
Nocedal, J., and Wright, S. (2006). Numerical optimization (Springer Science \& Business Media).

Rabiner, L. R., and Schafer, R. W. (1978). Digital processing of speech signals, 100 (Prentice-hall Englewood Cliffs, NJ).

Rienstra, S. W. (2005). "Webster's horn equation revisited," SIAM Journal on Applied Mathematics 65(6), 1981-2004.

Scheichl, S. (2004). "On the calculation of the transmission line parameters for long tubes using the method of multiple scales," in The Journal of the Acoustical Society of America $\mathbf{1 1 5}(2), 534-555$.

Schmutzhard, S., Chatziioannou, V., and Hofmann, A. (2017). "Parameter optimisation of a viscothermal time-domain model for wind instruments," in Proceedings of the 2017 International Symposium on Musical Acoustics, pp. 27-30.

Silva, F., Guillemain, P., Kergomard, J., Mallaroni, B., and Norris, A. N. (2009). "Approximation formulae for the acoustic radiation impedance of a cylindrical pipe," Journal of Sound and Vibration 322(1-2), 255-263.

Silva, F., Vergez, C., Guillemain, P., Kergomard, J., and Debut, V. (2014). "MoReeSC: a framework for the simulation and analysis of sound production in reed and brass instru- 
ments," Acta Acustica united with Acustica 100(1), 126-138.

Stinson, M. R. (1991). "The propagation of plane sound waves in narrow and wide circular tubes, and generalization to uniform tubes of arbitrary cross-sectional shape," The Journal of the Acoustical Society of America 89(2), 550-558.

Thibault, A., and Chabassier, J. (2020). "Viscothermal models for wind musical instruments," Inria Research Report, https://hal.inria.fr/hal-02917351.

Thompson, S. C., Gabrielson, T. B., and Warren, D. M. (2014). "Analog model for thermoviscous propagation in a cylindrical tube," The Journal of the Acoustical Society of America 135(2), 585-590.

Tijdeman, H. (1975). "On the propagation of sound waves in cylindrical tubes," Journal of Sound and Vibration 39(1), 1-33.

Tournemenne, R., and Chabassier, J. (2019). "A comparison of a one-dimensional finite element method and the transfer matrix method for the computation of wind music instrument impedance," Acta Acustica united with Acustica 105(5), 838-849.

Van Der Schaft, A. (2006). "Port-hamiltonian systems: an introductory survey," in Proceedings of the international congress of mathematicians, Citeseer, Vol. 3, pp. 1339-1365. 
Viscothermal time model for wind instruments

${ }_{446}$ Zwikker, C., and Kosten, C. W. (1949). Sound Absorbing Materials (Elsevier Publ. Comp.). 
Viscothermal time model for wind instruments 